\title{
Análisis estadístico del uso de los sistemas de mensajería electrónica en aparatos móviles ${ }^{1}$
}

\author{
Ramón Alberto Mendoza ${ }^{2}$ \\ UNITEC, Tegucigalpa, Honduras.
}

(Recibido: Diciembre, 2014 y Aceptado: Julio, 2015)

\begin{abstract}
Resumen:
Este trabajo de investigación muestra como los sistemas de mensajería electrónica (SME) están siendo utilizados cada vez más frecuentemente, por los usuarios de los servicios móviles. De igual forma se plantean las preferencias de los usuarios por los diferentes sistemas de mensajería electrónica disponibles actualmente y que aparatos móviles son los utilizados como medios para enviar y recibir mensajes de texto. La investigación también está destinada a determinar la preferencia de los usuarios a enviar un mensaje de texto en vez de hacer una llamada telefónica normal e incluye una apreciación del público para saber si los usuarios consideran que los SME, están sustituyendo la comunicación formal a partir de la opinión de si preferimos enviar un mensaje de texto o hacer una llamada. También obtenemos los tipos de dispositivos móviles que utilizan los usuarios para los SME. Para finalizar con la investigación, se presentan para que están utilizando los usuarios los diferentes SME.
\end{abstract}

Palabras Claves: Sistemas de mensajería electrónica, Sistemas móviles, Aparatos móviles, Mensajes de texto.

\begin{abstract}
:
This research shows how electronic messaging systems (EMS) are being used increasingly frequently by users of mobile services. It also considers the users' preferences for the different electronic messaging systems currently available and the types of mobile devices being used as the means to send and receive text messages. The investigation tries to determine users' preferences to send text messages instead of making a normal phone call. It includes a user's assessment to find out whether they think SME is replacing formal communication. The study also presents the types of mobile devices used for SME. To conclude the research, we present the findings on how people use SME.
\end{abstract}

Keywords: Electronic messaging systems, Mobile systems, Mobile devices, Text messaging.

\section{Introducción}

Desde el inicio de la humanidad, podemos ver claramente varias etapas que han marcado nuestra evolución, sin querer referirnos a esto, como la evolución definida por Charles Darwin (1809 - 1882) sino la evolución individual del ser humano, modificando la forma de ver las cosas, la forma de pensar, la forma de actuar y por supuesto la modificación de toda la sociedad.

Muchas de estas etapas, han logrado cambios significativos, no siendo el objeto de este documento mencionarlas una por una, pero si enfocarnos en una en particular que cambió la sociedad, la individualización del hombre para convertirlo en un ente parte de la sociedad mundial: La Revolución Industrial.

\footnotetext{
${ }^{1}$ Articulo ganador del Tercer Lugar en la categoría de Docentes de Pregrado del Concurso de Investigación UNITEC-CEUTEC 2014.

2 Autor para correspondencia. Email: ramon.mendoza@unitec.edu
} 
A inicios de La Revolución Industrial en Norte América (1790 - 1860), Merritt Roe Smith y Robert Martello mencionan (en mi propia traducción) que el Secretario del Tesoro Alexander Hamilton argumentó que: "no solo la riqueza, también la independencia y seguridad del país, aparecen conectadas con la prosperidad de las manufacturas.” Además se reconoce cómo la producción propia puede incidir en todos los aspectos de la sociedad, incluso en la seguridad y el bienestar de todos los individuos. Pero nuestro interés de mencionar la Revolución Industrial, es para recalcar el hecho de que el hombre ya no pertenece a una sociedad limitada, y reconoce la movilidad de los hombres, a su conveniencia llamados "mano de obra”, no solo dentro de un país o una región, sino una movilidad global.

Ahora podemos mencionar la Era Digital como otra época que ha influido o deberíamos decir está influyendo inmensurablemente en la formación del individuo, causando cambios en la forma de ver las cosas y por supuesto generando cambios en la sociedad, entendiendo esta, no como la sociedad local o regional, ya que estos cambios se están haciendo simultáneamente en todo el mundo.

Con relación a La Era Digital, sin decir cuando inició o cuánto durará; podemos observar los elementos que la caracterizan. Cuando mencionamos que nuestros niños y jóvenes son nativos digitales, es porque reconocemos su forma de actuar, cuando miramos una niña de menos de dos años, hacer pasar las pantallas en un iPad, iPhone o Samsung, para buscar una aplicación que ya sabe dónde está, que ya sabe que es lo que hace, y ya sabe que tiene que hacer para echarla a andar.

Cuando vemos un adolescente que en una mano tiene una Tablet, y en la otra tiene un celular "inteligente", que ha aprendido a escribir con ambas manos y con un solo dedo, más rápido de lo que aprendimos nosotros con tantos años de uso del típico procesador de palabras.... Estos pequeños pertenecen a una era, en la cual la mayoría de nosotros nos tenemos que adaptar, por lo que nosotros deberíamos ser llamados los migrantes en la Era Digital.

"Todos ellos son nativos digitales... Y todos ellos tienen las habilidades para usar esas tecnologías.” (En mi propia traducción) (Palfrey, John y Gasser, Urs, 2008)

John Palfrey y Urs Gasser continúan mencionando: "Son más propensos a enviar un mensaje instantáneo (IM) que a levantar el teléfono para concertar una cita más tarde ese mismo día...”.

Nosotros mismos a veces no somos capaces de notar lo apegados que nos encontramos a nuestros dispositivos móviles, mas con la intención de enviar mensajes o de esperar una respuesta o comunicación escrita, que para hacer o esperar una llamada de voz. ¿Deberíamos entonces en nuestro estudio, denominar a esto como una era, como la "era de las comunicaciones móviles”? Por supuesto, este es un sub-producto generado por la era digital. Pero ¿por qué debemos hablar de las comunicaciones móviles cuando nos interesan los sistemas de mensajería electrónica? Esto es lo que tratamos de determinar en nuestro estudio, consultando si los SME (sistemas de mensajería electrónica) se utilizan con más frecuencia en dispositivos móviles que en computadores o dispositivos fijos.

Es nuestro interés, determinar si los sistemas de mensajería electrónica son más usados a través de aparatos móviles, o si podemos entonces determinar si la era digital está más ligada a los sistemas fijos. Estos resultados los esperamos presentar en las conclusiones emanadas de las encuestas realizadas. 
Sin menospreciar la importancia, no se consideró en esta investigación discutir o exponer los dos puntos de vista respecto a las comunicaciones en la era digital: la generación que se comunica sin hablar y la generación más comunicada a través de los mensajes de texto. Estos dos puntos de vista sólo serán mencionados en caso de que afecten nuestras conclusiones.

Haremos un resumen sobre la validez de la muestra, y la aplicación a una población que se encuentra en Tegucigalpa, Honduras, con la confianza de que los resultados podrán extrapolarse al comportamiento de todo el país.

Finalmente presentaremos las conclusiones de los resultados obtenidos, así como las tabulaciones y gráficas que se mostrarán en los anexos. Podemos sustentar dichos resultados, aclarando que las conclusiones, son opiniones emanadas de la agrupación estadística de los resultados, pero emitidas por el autor.

\section{Marco teórico}

\subsection{Las telecomunicaciones y los mensajes de texto.}

Gonzalo Ortiz Crespo en Diario Hoy (01 de Junio del 2014), menciona el artículo de Mark Prensky: Digital Natives, Digital Immigrants: “... los estudiantes de hoy en escuelas y colegios, son personas que han cambiado radicalmente; para quienes el sistema educativo ha quedado obsoleto.”

Encontramos gran cantidad de libros, artículos y discusiones respecto a la forma de aprender, forma de enseñar, forma de expresarse y en general, la forma de comunicarse en esta llamada "era digital”. La finalidad es siempre la comunicación entre una persona y otra, o entre un grupo de personas. Si ese objetivo se logra, ¿Cómo podremos determinar si el uso de una tecnología es mejor que el uso de otra? ¿Podremos medir los niveles de comunicación lograda?

No siendo este mi campo de acción ni el interés de este estudio, creo conveniente dejar esto para temas futuros, para exponer ideas que hemos encontrado sobre dos sistemas fundamentales de comunicación: la comunicación remota oral o formal y la comunicación remota mediante mensajes de texto o lo que hemos llamado en este documento Sistemas de Mensajería Electrónica (SME). Es muy común que a estos sistemas de mensajería se les catalogue como Sistemas de Mensajería Instantánea (IM), pero para el presente estudio, he preferido la denominación primera, por las implicaciones que puede tener el uso del término “instantánea”.

Es tema de discusión permanente, si la comunicación se debe realizar teniendo la posibilidad de escuchar los tonos de voz e incluso si la comunicación se mejora, cuando tenemos la posibilidad de ver gestos que nos ayuden a que la comunicación sea más efectiva.

Las telecomunicaciones han propiciado las comunicaciones escritas desde el siglo XVII con el uso del telégrafo por Samuel Morse (1791 - 1872) hasta las que hoy en día podemos incluir dentro de los que en este documento llamamos SME. Podemos observar que la invención del Teléfono por Alexander Graham Bell (1847 - 1922) quien consiguió la patente en Estados Unidos de América en 1876, se realizó tiempo después, y debo mencionar al inventor Italiano Antonio Meucci (1808 - 1889), quien fue reconocido como el inventor del aparato telefónico el 11 de junio de 2002.

Meucci construyó el primer modelo de aparato telefónico en 1855 y en 1871 presentó un modelo perfeccionado. Y si comparamos la presentación de la primera operación de un 
telégrafo por Joseph Henry (1797 - 1878) en 1829, podemos remontarnos a pensar que las comunicaciones a distancia a través de mensajes cortos fueron los pioneros de las telecomunicaciones; y en efecto así fue.

Pero por la época, y por el desarrollo galopante de las tecnologías orientadas a las comunicaciones de voz durante los siguientes años, son estas, a través de las telecomunicaciones las que prevalecen. Desde 1877 que se tiene conocimiento fue la invención del primer conmutador manual para llamadas telefónicas, en 1892 Almond B. Strowger (1839 - 1902) construyó el primer conmutador telefónico automático. Desde entonces la tecnología ha evolucionado hasta los últimos desarrollos de conmutadores IP, capaces de conmutar voz o paquetes de datos e internet sobre una misma plataforma.

\subsection{Los sistemas de mensajería electrónica (SME)}

De los sistemas de mensajería electrónica utilizados actualmente, solo el denominado Servicio de Mensajes Cortos (SMS) es el que ha sido regulado por estándares internacionales, según se menciona adelante. Los demás sistemas de mensajería utilizados han aparecido como aplicaciones, desarrolladas como parte de las redes sociales y para ser instaladas en un aparato móvil o en la PC, laptop o tablet.

\section{$\underline{\text { Servicio de Mensajes Cortos (SMS) }}$}

Podemos observar que los Sistemas de Mensajería Electrónica se usan masivamente desde el lanzamiento de las plataformas móviles en su Segunda Generación (2G), con la tecnología GSM. Esto se menciona en varios textos y artículos cuando hacen ver que el Servicio de Mensajes Cortos (SMS por sus siglas en inglés) fue considerado un fenómeno excepcional por los proveedores de servicios. De hecho, "la cantidad de aparatos de celulares creció 12,6\%, llamadas un 29,3\% y los SMS un 22,4\% anual” este crecimiento es referenciado al año anterior.” (Universidad Nacional de Lomas de Zamora, 2010)

En los estándares ETSI (European Telecommunication Standard Institute) que en muchos casos son la base para los estándares adoptados por la ITU (International Telecommunications Union), Normativa ETS 300536 (Octubre 1996), se establece las condiciones técnicas del Sistema de Telecomunicaciones Celulares Digitales para proveer el Servicio de Mensajes Cortos (SMS). La principal característica es que los mensajes se definen como mensajes "punto a punto". Otra característica importante es que el tamaño del mensaje no puede ser mayor a 160 caracteres de 7 bits.

Esta característica en un inicio, fue considerada una limitante para que el SMS llegara a popularizarse, pero al poco tiempo se vio que los usuarios aprendieron a manejar esta limitante muy bien y usaron cada vez más el SMS. Desde mi punto de vista, esta limitante se puede relacionar con la que se impuso al servicio de telegrafía (5 palabras).

En el informe del ITU “The World in 2010”, (Traducción libre del inglés al español por el autor) se menciona que el número total de SMS enviados globalmente se ha triplicado entre el 2007 y 2010, de un estimado de 1.8 billones (nomenclatura del Sistema Métrico Decimal) a 6.1 billones de mensajes. En otras palabras, cerca de 200,000 mensajes de texto son enviados cada segundo.

El mismo reporte de la ITU, mencionado arriba, hace una estimación que el mercado de SMS tiene un valor aproximado de USD 812,000 cada minuto (o aproximadamente USD 14,000 cada segundo). Y que en el 2009 los ingresos provenientes de SMS fueron cerca del 12\% del ingreso total del mayor operador de telecomunicaciones de China. Esto nos da una idea de la importancia de SMS en el mercado global de las telecomunicaciones. 
Hoy en día, vemos que el fenómeno ha cambiado, pero los sistemas de mensajería hoy utilizados, reconocen que su idea original se basó en el primero de los sistemas de mensajería: SMS.

\section{El servicio de mensajería por BlackBerry (BBM)}

El servicio de Mensajería Electrónica propietario de la compañía Canadiense Blackberry Limited (BBRY LTD) para sus teléfonos inteligentes se consideró un sistema revolucionario de mensajería electrónica, por las condiciones de seguridad, opciones de habilitar servicios corporativos y otros, que influyeron para su adopción por el mercado masivo, pero en particular por el mercado corporativo. Este fue el primero de los SME que ya no fueron incluidos en recomendaciones o estándares internacionales.

Las características principales de BBM para atraer usuarios fue dar a estos una forma fácil y rápida de enviar y recibir mensajes instantáneos usando sus códigos de PIN (y no sus números celulares). Esta característica fue considerada muy importante por los usuarios, aun tiempo después de la aparición de otros sistemas como WhatsApp, que basaron su comunicación en el número celular. Este servicio es gratis y los usuarios pueden además llevar sus correos electrónicos y varias conversaciones al mismo tiempo. "Tiene también la opción de chat en grupo, enviar y recibir archivos, ver y enviar contactos y hacer búsquedas del estado del mensaje” (Dannenfelt, D., 2006).

Consultando “BlackBerry Messenger User Guide”, se pueden obtener de forma detallada, todas las característica del servicio BBM, pero las dos principales ventajas para uso corporativo, fueron las que lo convirtieron como una opción para uso masivo, estas características son: Que los usuarios solo pueden mandar mensajes a otros usuarios si conocen su código PIN y la otra característica fue que el servicio BBM solo se podía establecer entre dos usuarios que tuvieran aparatos móviles BlackBerry.

BlackBerry, desde inicios de 2013 a la fecha ha hecho intentos para mantenerse en el mercado; entre ellas lanzamiento de aparatos móviles BlackBerry rediseñados en categoría de "smartphones", hacer que BBM esté disponible como aplicación para Android y iPhone, para que los usuarios con aparatos BlackBerry puedan ampliar su base para interactuar con otros usuarios que no tienen el mismo dispositivo móvil; pero creo de forma personal que no han resultado, porque no ha logrado competir contra la fusión Microsoft-Nokia y la predominancia en el mercado de los teléfonos móviles de Samsung y iPhone.

\section{WhatsApp}

No puedo por ahora afirmar, si WhatsApp fue el primero de los sistemas de mensajería electrónica en entrar al ruedo y a la competencia con los sistemas de mensajes cortos existentes: BlackBerry y SMS. Lo que sí puedo afirmar es que es el SME que más a dado que hablar, de todos los SME que se compararán en esta investigación. Podemos encontrar una definición de sí misma en http://www.whatsapp.com/ (sitio oficial de la compañía) o en http://es.wikipedia.org/wiki/WhatsApp que nos mencionan que "la aplicación WhatsApp fue creada en Enero del 2009, y que su segunda versión - junio 2009 - logró tener 250,000 usuarios”.

Desde su inicio, la aplicación WhatsApp fue diseñada para dispositivos móviles, y no para desktops o laptops, por lo tanto, no se consideró competencia de los sistemas de mensajes de Facebook o Google. Pero su creciente uso se evidenció desde un inicio, y podemos referirnos a artículos y publicaciones recientes sobre WhatsApp en los que encontramos menciones como "Los responsables de WhatsApp aseguran que siete de 
cada diez usuarios utilizan a diario su servicio e intercambian cada día unos 50,000 millones de mensajes.” (Suárez, E., 20 de Febrero de 2014).

Todo lo anterior nos sirve para mencionar el crecimiento exponencial en el uso de las aplicaciones que ofrecen los servicios de mensajería electrónica a través de sistemas o aparatos móviles. ¿Cómo ha logrado WhatsApp tanta popularidad? Considero que su popularidad está fundamentada en sus características, desde su fácil instalación a su simplicidad de uso y por supuesto a lo ya mencionado antes, la intercomunicación entre dispositivos de diferentes fabricantes. Sus creadores han reconocido que se fundamentaron en los sistemas anteriores de SMS y BBM, para crear sus características primarias, pero son las funciones adicionales y simplicidad las que le han hecho posicionarse entre las aplicaciones más utilizadas por los usuarios de todo el mundo.

\section{Alcance de la investigación}

\subsection{Enunciado del problema}

Cada vez es más frecuente que nos encontremos con personas, que estando solas, en el trabajo, en alguna ocupación y aun estando reunidas para un fin común (trabajo, social o para compartir entre ellas), están mirando su teléfono móvil y haciendo alguna actividad en su teléfono móvil. Suponemos que en la mayoría de los casos, estas personas están utilizando su celular para enviar o recibir mensajes de texto utilizando alguno de los sistemas de mensajería electrónica disponibles y que se incluyen en esta investigación. Si las personas estando reunidas con otras personas, están comunicándose o queriendo saber de otras personas y; en caso extremo (a jucio del autor, que he podido observar en varias ocasiónes), que aun estando frente a frente o en el mismo espacio físico, dos personas o más se están comunicando vía mensajes de texto. Esto nos llevó a planteamos la hipótesis: Las personas están hoy en día prefiriendo la comunicación por mensajes de texto electrónico versus la comunicación formal mediante conversación hablada o escrita (correo).

En este momento no definiremos esto como una situación perjudicial para la comunicación entre las personas, solo trataremos de determinar tendencias sobre el uso de los sistemas de mensajería electrónica para comunicarse.

\subsection{Planteamiento del problema}

Trataremos de indagar lo que consideran nuestros encuestados sobre este tipo de comunicación a través de mensajes de texto. Esperamos que los resultados nos arrojen las tendencias del uso de SME para la comunicación (no se discutirá si la comunicación es efectiva o inefectiva o impersonal).

\subsection{Preguntas de investigación}

- ¿ ¿A qué grupo poblacional pertenece? (edad, educación/ laboral)

- ¿ ¿Prefiere Ud. enviar un mensaje de texto a hacer una llamada?

- En su opinión ¿Los SME pueden llegar s sustituir la comunicación formal?

- ¿En qué se utilizan más frecuentemente los SME?

- ¿ ¿Qué tipo de dispositivos utiliza para enviar y recibir mensajes de texto? 


\subsection{Objetivos de la investigación}

\section{Objetivos generales}

Determinar si la población considera que los SME tienden a sustituir las comunicaciones formales escritas o de voz.

Determinar cuáles son los sistemas de mensajería electrónica más utilizados, según grupos de edades y nivel de educación o trabajo.

\section{Objetivos específicos}

1. Aplicar una encuesta en un grupo poblacional divergente, para tener resultados que se puedan extrapolar a toda la República de Honduras.

2. Determinar cuál es el SME preferido por la población de Honduras y cómo es utilizado.

3. Determinar cuáles son los aparatos móviles y/o tablet preferidos por la población encuestada y si los SME son utilizados desde este tipo de aparatos móviles.

4. Determinar si los sistemas SME se están utilizando para comunicaciones con amigos, con familiares, para asuntos de trabajo o de educación; o para difusión de mensajes de mercadeo o anuncios publicitarios.

En la Tabla 1. Se detallan las que se consideraron como variables de investigación.

Tabla 1. Variables de investigación

\begin{tabular}{|l|l|l|}
\hline \multicolumn{1}{|c|}{ Variable } & \multicolumn{1}{|c|}{ Definición } & \multicolumn{1}{|c|}{ Indicadores } \\
\hline Grupo poblacional encuestado & $\begin{array}{l}\text { Representa el grupo } \\
\text { encuestado, determinando los } \\
\text { niveles de edades y de } \\
\text { educación o laboral. }\end{array}$ & $\begin{array}{l}\text { 1. Cantidad de encuestas por } \\
\text { grupos de edades. } \\
\text { Cantidad de encuestas por } \\
\text { niveles educativos y laborales. }\end{array}$ \\
\hline SME preferidos por la población & $\begin{array}{l}\text { Es la preferencia de la } \\
\text { población a utilizar uno u otro } \\
\text { SME. }\end{array}$ & $\begin{array}{l}\text { 1. Cuáles son los SME preferidos } \\
\text { por la población. }\end{array}$ \\
\hline $\begin{array}{l}\text { Preferencia de usos de aparatos } \\
\text { móviles }\end{array}$ & $\begin{array}{l}\text { Si los SME son utilizados desde } \\
\text { aparatos móviles, cual es la } \\
\text { preferencia de fabricante, según } \\
\text { grupo poblacional y nivel de } \\
\text { educación. }\end{array}$ & $\begin{array}{l}\text { 1. Preferencia de uso de aparato } \\
\text { móvil según niveles de edad y } \\
\text { educativo/ laboral. } \\
\text { Si los SME se utilizan desde } \\
\text { aparatos móviles o desde } \\
\text { tablets. }\end{array}$ \\
\hline $\begin{array}{l}\text { Sustitución de las comunicaciones } \\
\text { formales por los mensajes de texto } \\
\text { utilizando SME }\end{array}$ & $\begin{array}{l}\text { Apreciación de la población si } \\
\text { los SME tienden a sustituir los } \\
\text { sistemas formales de } \\
\text { comunicación. }\end{array}$ & $\begin{array}{l}\text { 1. Cantidad de población que } \\
\text { opina que los SME tienden a } \\
\text { sustituir los sistemas formales } \\
\text { de comunicación. }\end{array}$ \\
\hline
\end{tabular}

\subsection{Justificación}

Actualmente las tecnologías digitales han propiciado el uso de los SME y han revolucionado la forma de comunicación, y particularmente han tenido impacto en las telecomunicaciones incrementando la comunicación y eliminando las fronteras políticas, logrando que la comunicación sea efectiva no importando la ubicación de los interlocutores. Ante este contexto global, se podría pensar que el acceso a las tecnologías de comunicaciones móviles ha logrado influir sobre las formas de comunicación entre los individuos o grupos de individuos, sean esto para interés personal o para interés empresarial o corporativo. 
Metodológicamente la investigación propone un reto importante por carecer de información suficiente en la materia, es por esto, que para su validez se plantea el uso de información extraída directamente de fuente primaria (de los usuarios de los servicios SME) y metodología de análisis de información cuantitativa (análisis de los resultados de encuestas). Los resultados de la presente investigación ofrecerán cifras sobre la opinión de la población sobre el uso de los SME, sobre cuáles son los sistemas de mensajería preferidos; este tipo de estudio puede ser utilizado para definir políticas gubernamentales o regulatorias, aspectos legales y puede usarse por las empresas proveedoras de servicios móviles para ayudar a plantear nuevas estrategias, no solo respecto al uso de los sistemas de mensajería electrónica sino también respecto a brindar servicios o productos especiales que atiendan las necesidades de los usuarios; esto puede aplicarse en Honduras o en toda la región centroamericana, ya que es una región donde el acceso a los servicios móviles se está incrementando de manera exponencial.

\section{Metodologia}

\subsection{Determinación del tamaño de la muestra}

Con la finalidad de determinar el tamaño de la muestra, se tomó como base a: Levine, David M., Krehbiel, Timothy C., Berenson, Mark L. (Cuarta Edición. 2006) y a Fuller, Wayne A. (2009).

Se consideró el factor de proporción poblacional $\pi$ de forma tal que el factor $\pi \cdot(1-\pi)$ fuera el máximo posible que se da cuando $\pi=0.5$. El nivel de confianza deseado se consideró del $95 \%$, resultando el factor $Z=1.96$. El valor considerado inicialmente, para el error en los datos levantados fue de: $9 \%$

Utilizando lo establecido en el texto mencionado, la muestra a considerar debió ser:

$$
n=\frac{Z^{2} \cdot \pi(1-\pi)}{e^{2}} \cong 119
$$

Con el valor de muestras requeridas, obtenida arriba, según los textos, se procedió a generar las encuestas, teniendo un total de 164 encuestas válidas. Con estas 164 encuestas, se obtuvieron los resultados que se mencionan en el capítulo siguiente. Y con 164 muestras el error permitido ahora es:

$$
n=Z \sqrt{\frac{\pi \cdot(1-\pi)}{e^{2}}}=(1.96) \sqrt{\frac{0.25}{164}}=0.0765
$$

Como contribución se hicieron los cálculos, de cuanto representa el incremento en el error a considerar, cuando se eligen muestras superiores, y cuál será el comportamiento de la relación $\frac{e}{Z}$ para estudios posteriores.

Para los cálculos del margen de error en los resultados se tomó en cuenta a Triola, Mario F, (2010), y estos se muestran para los resultados más relevantes de la investigación. Mediante lo cual podremos afirmar que los resultados obtenidos en cada una de las consultas realizadas son válidos con los errores mostrados, puesto que los métodos de la encuesta producen muestras que pueden considerarse aleatorias simples y los ensayos son independientes. 
Tabla 2. Valores de confianza y error según para diferentes tamaños de muestras.

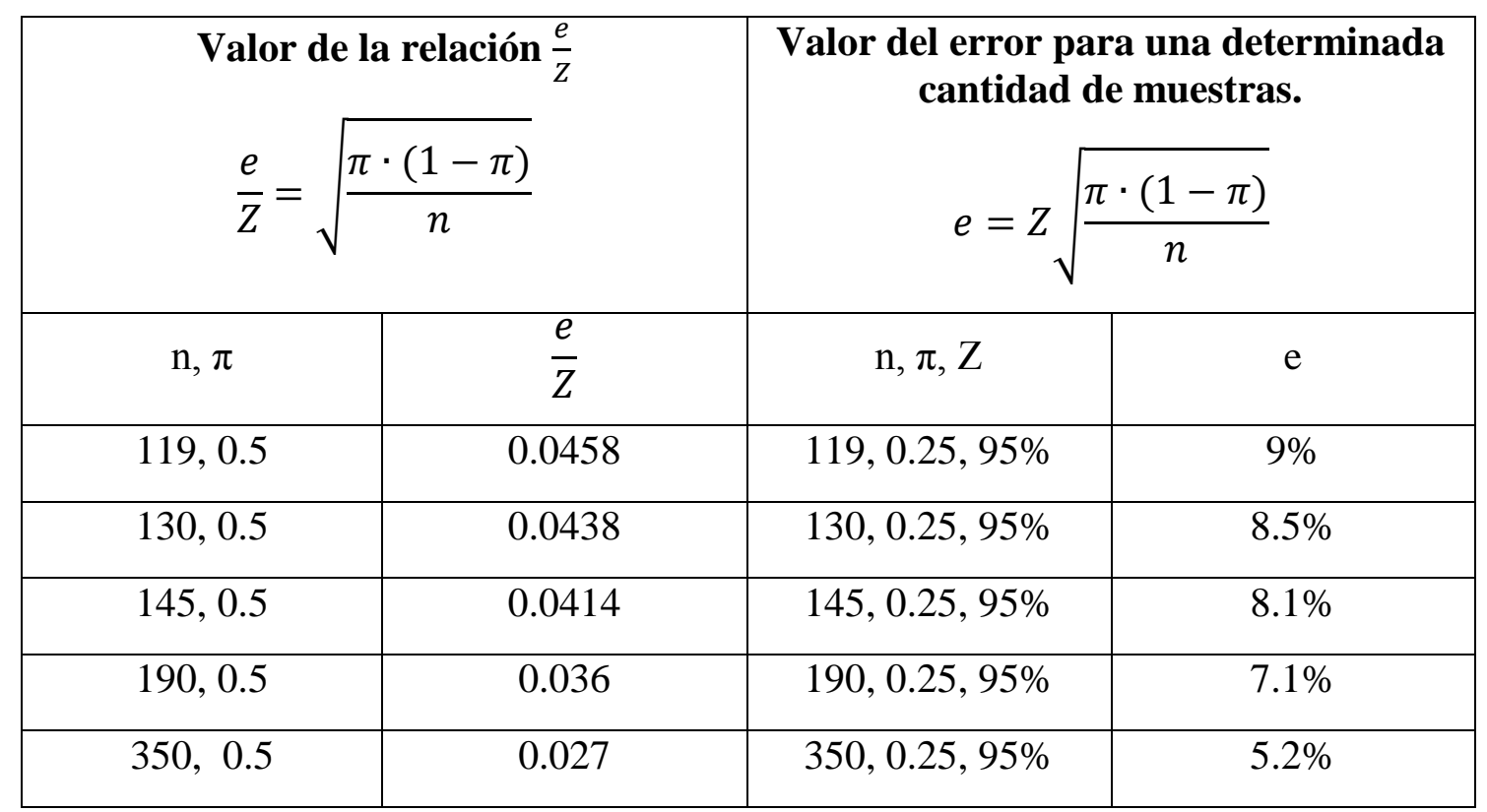

\subsection{Instrumento utilizado}

La investigación se realizó mediante encuesta directa la cual fue practicada por dos grupos de estudiantes de ingeniería en telecomunicaciones de UNITEC y como se mencionó en el marco teórico, se utilizó el software SPSS para la tabulación y gráficas.

\subsection{Tabulación y gráficas de las variables analizadas}

Para realizar la Tabulación y Gráficas se empleó el software SPSS (Statistical Product and Service Solutions), disponible en UNITEC, con la colaboración en el levantamiento de encuestas de estudiantes de UNITEC de ingeniería en Telecomunicaciones. A continuación se presentan los resultados relevantes que se obtienen a partir de las preguntas de investigación, los resultados se presentan gráficamente, para dar una idea total del resultado de la opinión respecto a la variable analizada.

\subsection{Comparación cuando el encuestado tiene muchas opciones.}

Debido a que la encuesta consideró la posibilidad de que un usuario utilizara más de un SME, se dio la oportunidad de valorar su preferencia en números, y se pidió que numerara del uno al tres según su uso, cuál es su SME preferido; siendo el 1 el de mayor preferencia y el 3 el de menor preferencia.

Esta diversidad en la valoración de los SME, resultó en que se definió para este estudio dos formas de análisis de la preferencia:

1. Factor de Uso Ponderado (suma ponderada promedio): se definió SPP como el valor ponderado de los resultados de cada selección multiplicados por el peso de dicha selección; entre el valor total de los resultados marcados.

2. Total de preferencia a cualquier nivel: Este factor de evaluación, suma las preferencias indicadas por los encuestados, no importando que nivel de preferencia marcaron. 


\section{Resultados de la investigación}

En la Figura 1, se puede observar que el $83 \%$ de las encuestas fueron aplicadas a una población entre los 19 y 30 años, lo que nos lleva a decir que las opiniones vertidas son el pensar de los jóvenes.

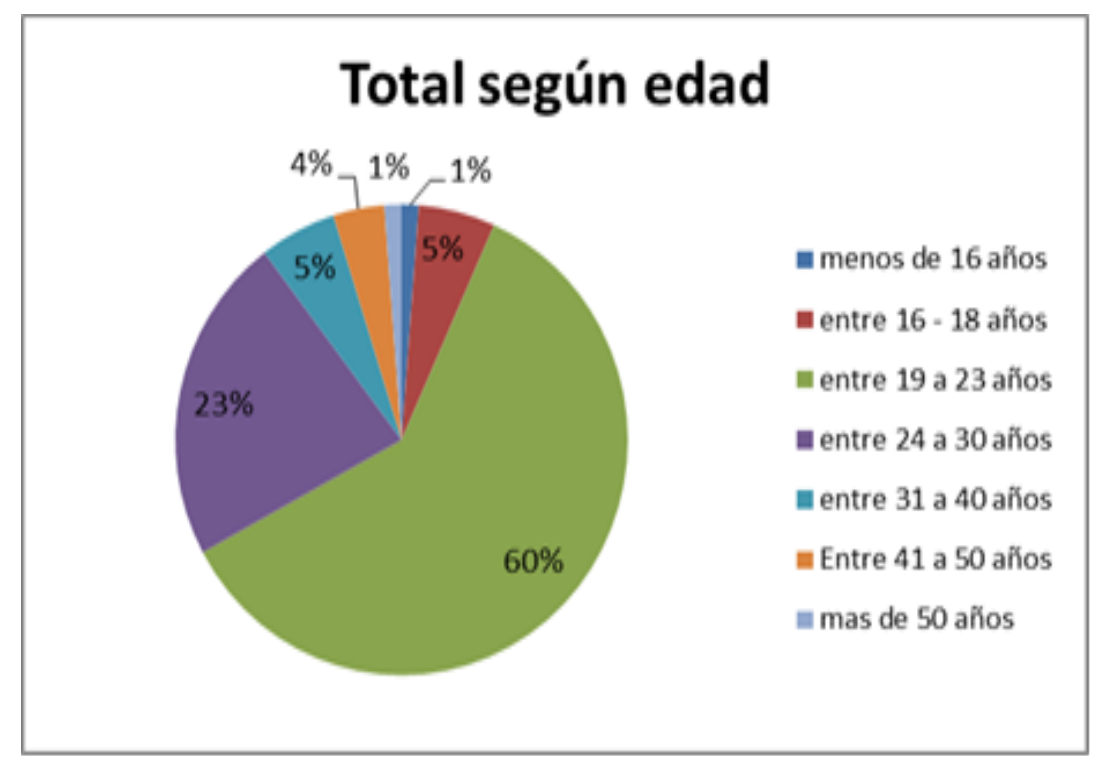

Figura 1. Grupo poblacional encuestado

En la Figura 2, se puede observar que el 94\% de las encuestas fueron aplicadas a personas de nivel educativo estudiantes universitarios, egresados universitarios y profesionales universitarios ya laborando.

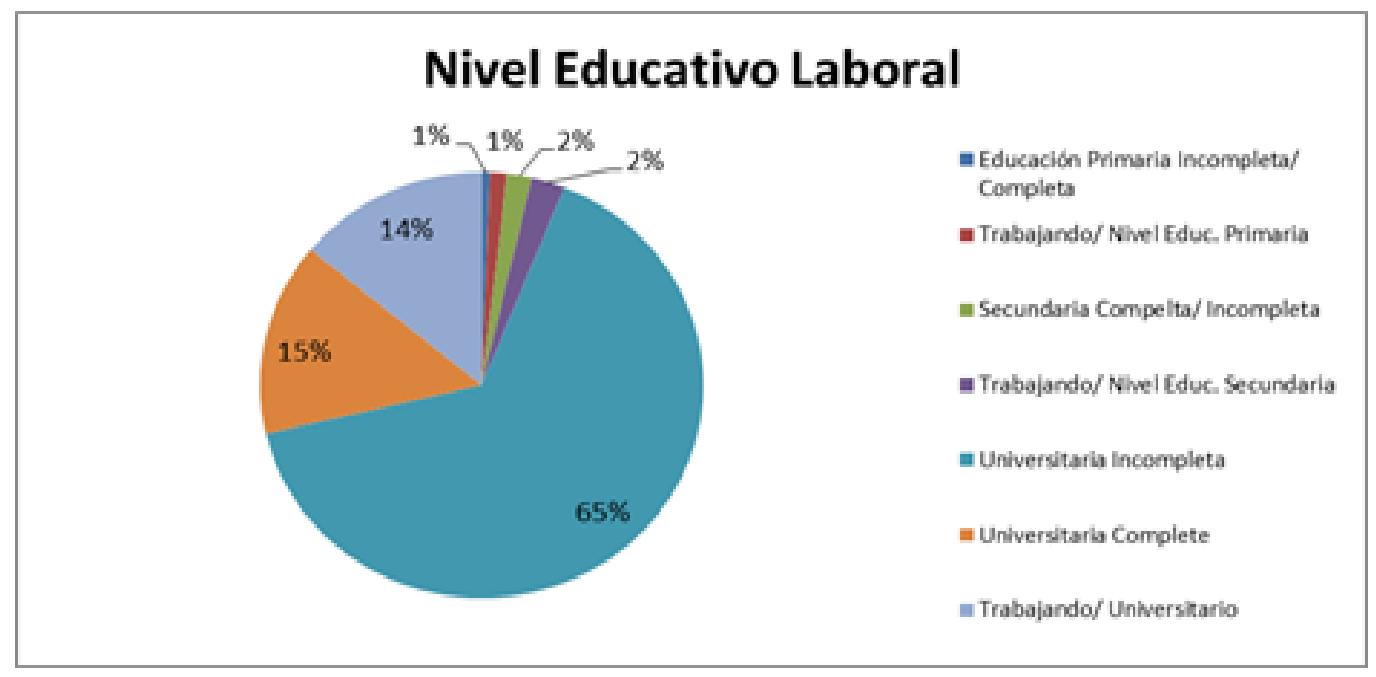

Figura 2. Nivel educativo de la población encuestada

\subsection{Sustitución de la comunicación formal}

En la Figura 3, se refleja la opinión si considera el encuestado que los mensajes de texto están sustituyendo de alguna manera a la comunicación formal, que según las consideraciones establecidas podemos decir que estos datos son ciertos con un margen de error del 5.8\%. Si sabemos que la población encuestada en su mayoría son jóvenes, es de mucha relevancia y por eso se consideró como el resultado mas importante, el hecho de 
que a pesar de que tienden a utilizar cada vez mas los SME, si consideran en un 82\% que se está sustituyendo la comunicación formal, por los mensajes de texto.

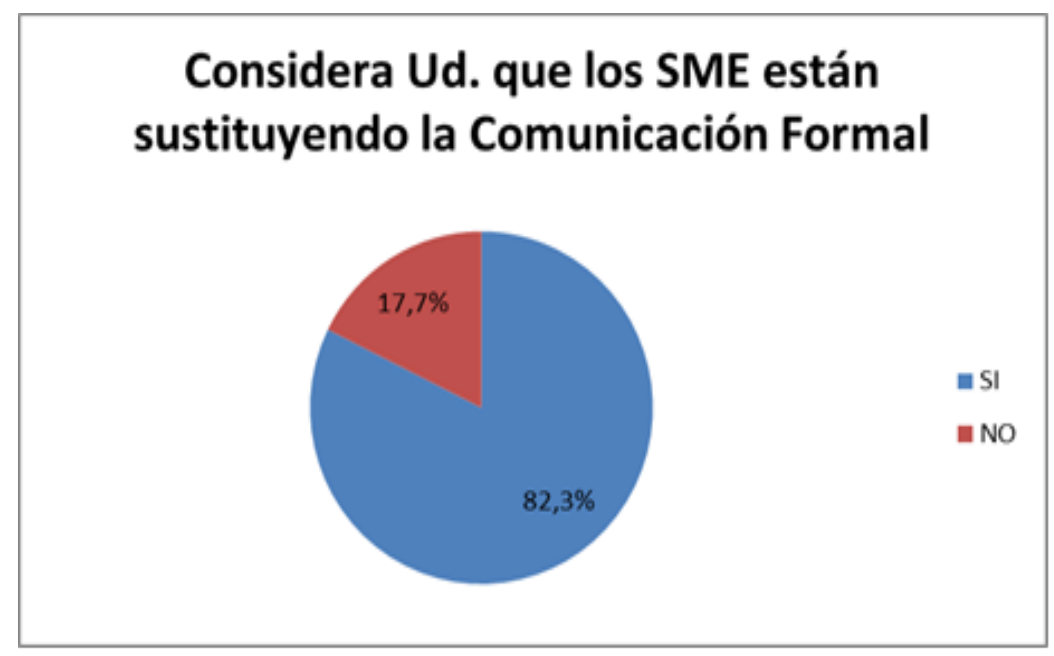

Figura 3. Opinión sobre si los mensajes están sustituyendo la comunicación formal

Es pertinente comentar que, según se muestran los resultados en la Figura 4, aunque la preferencia de enviar un mensaje de texto en vez de hacer una llamada se ubicó en un porcentaje de respuesta del $73.8 \%$, el $26 \%$ restante, que opinó que prefiere hacer una llamada, la totalidad de los mismos mencionan que hacen la llamada cuando quieren o necesitan una respuesta inmediata. El error de estimación para estos datos fue del 6.7\%.

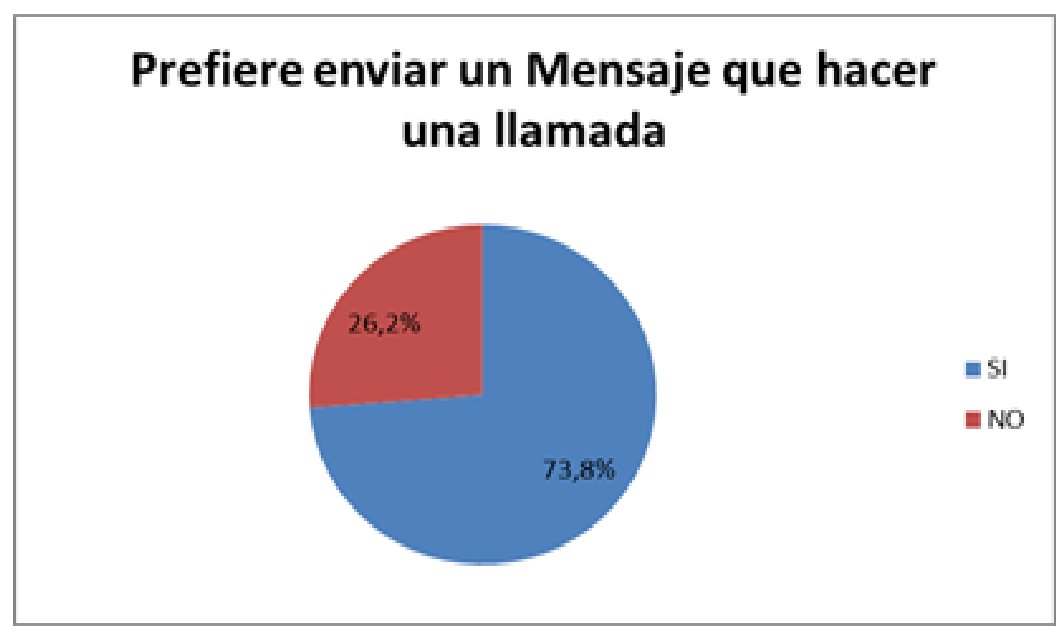

Figura 4. Preferencia de hacer llamada versus enviar un mensaje de texto.

Se considera pertinente comentar que, aunque la preferencia de enviar un mensaje de texto en vez de hacer una llamada se ubicó en un porcentaje de respuesta del 73.8\%, y al ver que los que consideran que los SME están sustituyendo la comunicación formal fueron un 82.3\%, nos indica que existe una opinión generalizada sobre la sustitución de la comunicación formal (hablada o escrita) para dar paso a la comunicación via cualquier sistema SME. 


\subsection{Preferencia de los diferentes SME}

\section{Evaluación según Suma Promedio Ponderada (SPP)}

Según se definió en el marco teórico, para tener un nivel de comparación entre tantas opciones que tenía el encuestado, para decidir su preferencia de tipo de SME que más usa, y que de cada uno podría indicar entre tres niveles de uso, se definió el SPP como mejor indicador comparativo.

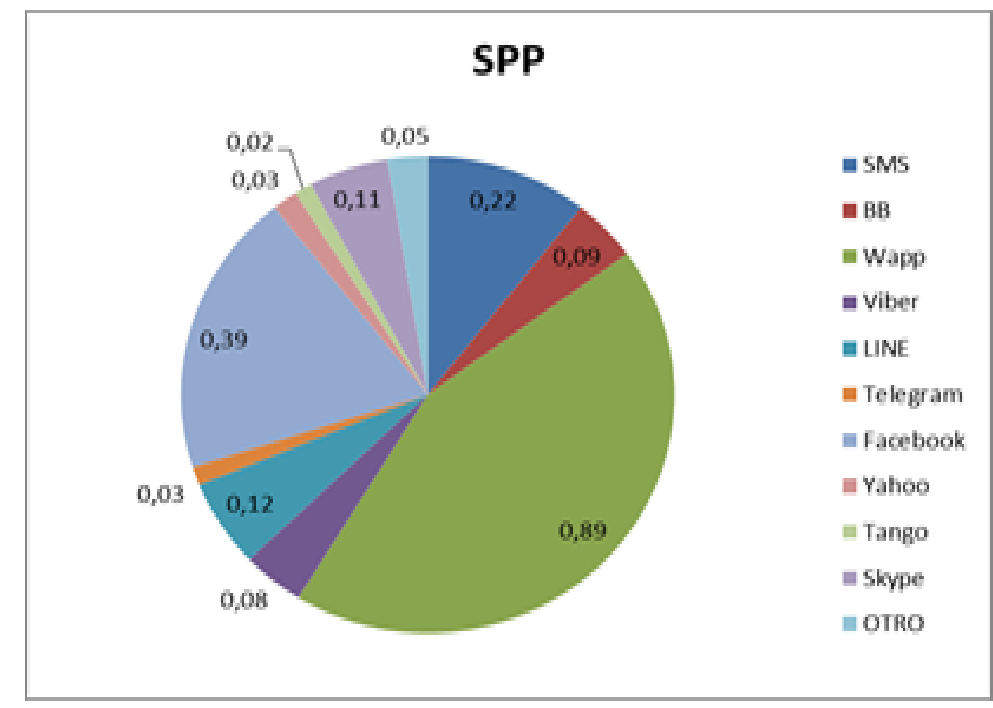

Figura 5. Comparación según SPP de preferencia del uso de los SME

Evaluación según el total de preferencia en cualquier nivel

Como complemento a las comparaciones mostradas en la Figure 5; se presentan los resultados de la Figure 6, que son obtenidos mediante la suma de todos los encuestados que marcaron ese SME como preferencia.

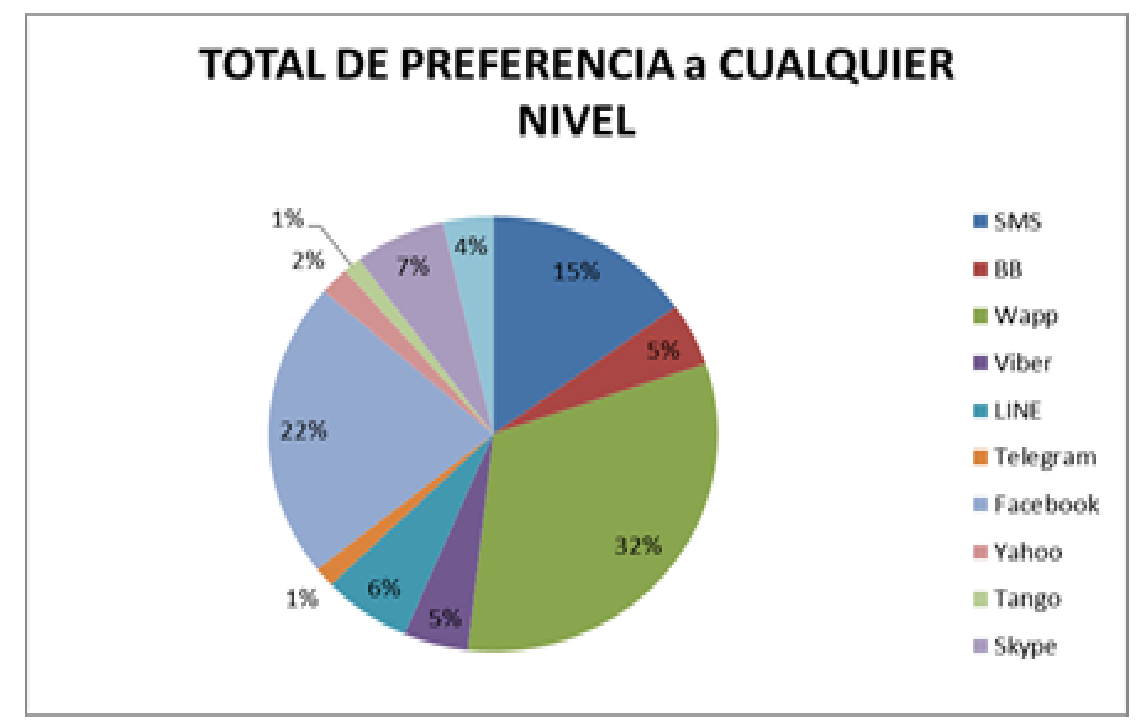

Figura 6. SME marcado como preferente, en cualquier nivel de preferencia

En esta gráfica solo nos dá el total de marcas que tuvo un tipo de SME, pero puede observarse que conserva la misma tendencia que la medición planteada a través de SPP. 


\subsection{Preferencia de uso de aparatos móviles}

Preferencia global de uso de aparatos móviles

Para obtener una preferencia sobre que aparatos se están empleando para el envío de los SME, se pidió a los encuestados responder las preguntas sobre su fabricante de aparato móvil, así como el fabricante de su otro sistema utilizado con los SME.

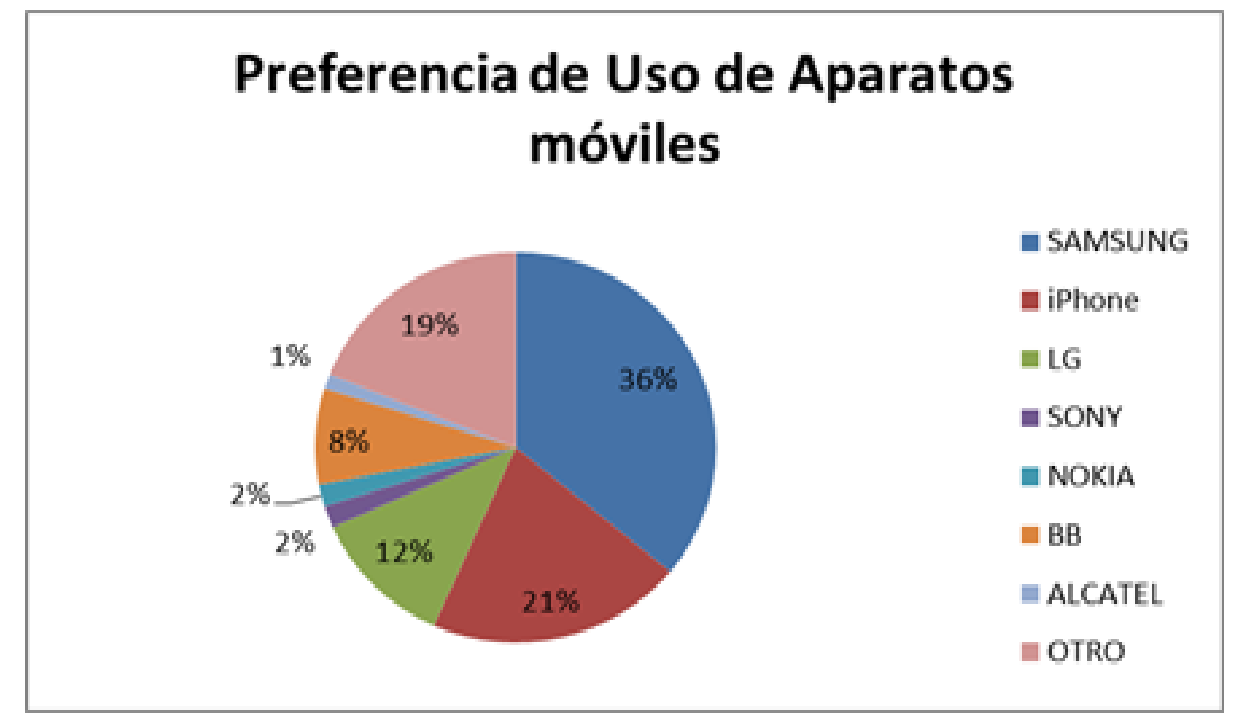

Figura 7. Fabricante de su apartato móvil, en el cual usa su SME.

En la Figure 7, se muestran los resultados de preferencia según fabricante de aparatos móviles, resultando una preferencia global de 36\% para Samsung contra un 21\% de preferencia para iPhone.

Preferencia de aparatos móviles según grupo poblacional

En la Figure 8, se puede notar claramente que la preferencia para todos los grupos poblacionales encuestados, el fabricante Samsung tiene una preferencia superior, y en segundo lugar de preferencia se ubica el fabricante iPhone.

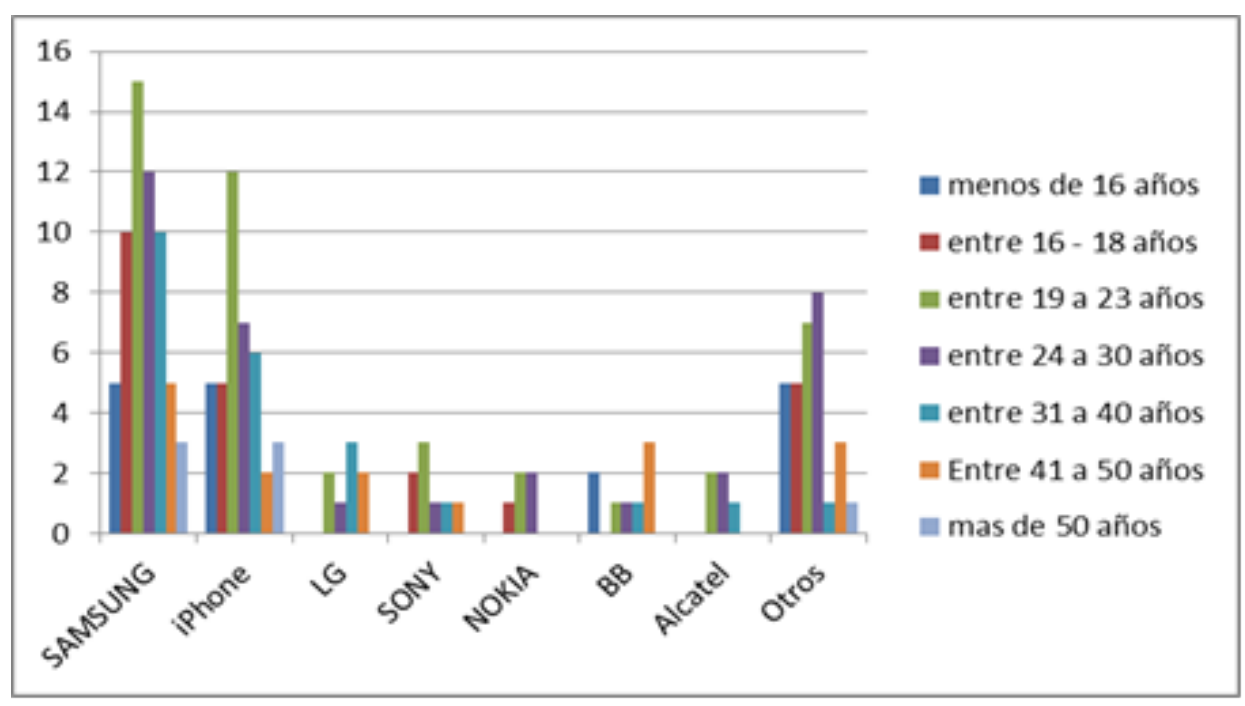

Figura 8. Preferencia se aparatos móviles según grupo poblacional 
Es importante resaltar que las Tablet más utilizada es la iPad con un 55\% de uso, contra un 25\% de personas que usan una Samsung; estos datos son mostrados en la Figure 9.

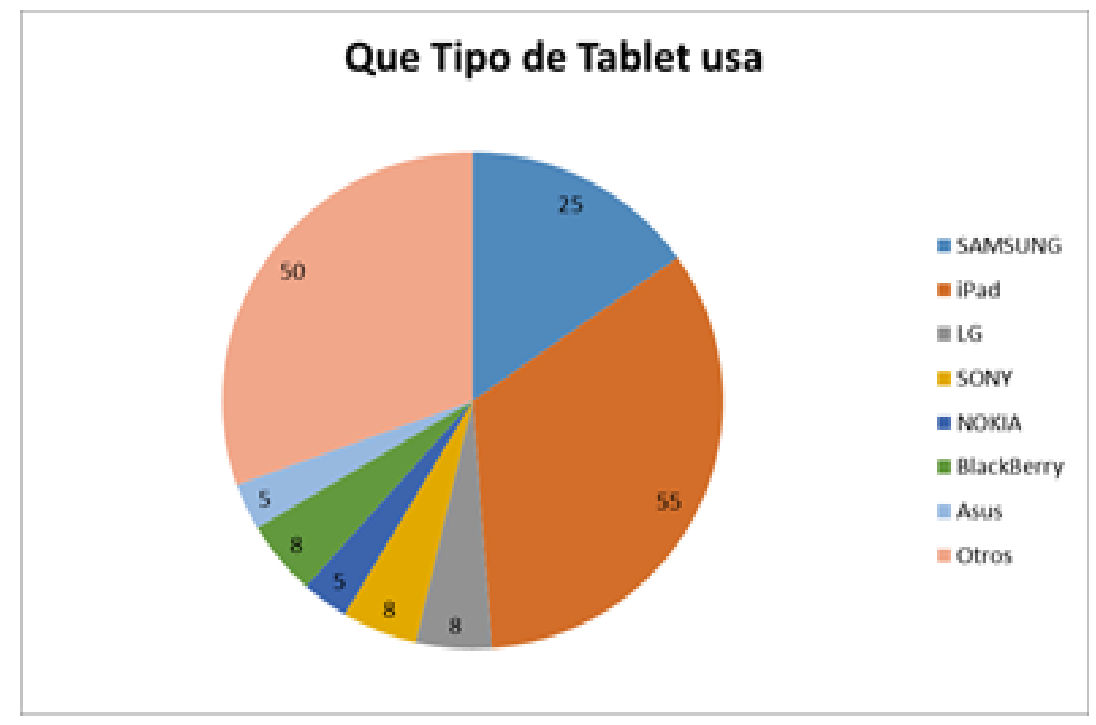

Figura 9. Tipo de dispositivo tipo Tablet que utiliza.

\section{4. ¿Para qué se utilizan los SME?}

Considerando la posibilidad de que el uso de los SME, fuese para otras opciones diferentes a comunicarse con amigos, se presentaron las opciones de: a) amigos, b) familiares, c) trabajo o estudios, d) publicidad y d) otros.

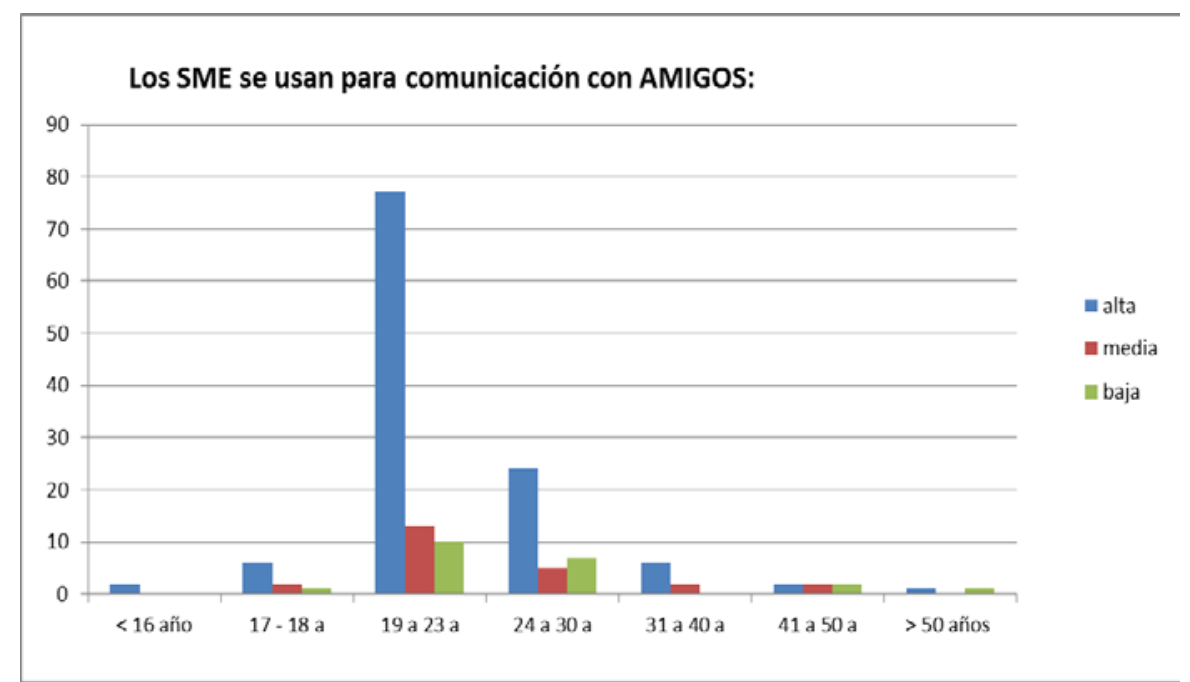

Figura 10. Preferencia de uso de los SME, para comunicarse con AMIGOS

En todos los rangos de edades y grupos educacionales, coinciden en que utilizan los SME para comunicaciones principalmente con amigos, en la Figura 10 se muestra el nivel de preferencia, primero para todos los rangos de edades consultados. Los resultados de sus preferencias de uso para con familiares y por asuntos laborales, se ubican muy por debajo de la preferencia de uso número uno que es: amigos. 


\section{Conclusiones}

\subsection{Se está cambiando la comunicación formal para utilizar mensajes de texto}

$\mathrm{Al}$ analizar que del total de la población encuestada, un 82.3\% considera que los mensajes de texto si están cambiando la forma de comunicación, de una formal (hablada y escrita) a una comunicación vía teléfonos móviles y mensajes de texto; esto es importante para considerar en muchos aspectos de la sociedad: desde la forma de comunicarnos con nuestros amigos, familiares o por asuntos de trabajo, los niveles de atención que pueden prestar las nuevas generaciones a la comunicación hablada en comparación con la comunicación vía mensajes de texto, los sistemas legales y el sistema de educación.

Todos estos estos datos sugieren que los enfoques de los aspectos mencionados pueden ir variando con el tiempo, en especial si se considera que la tendencia es a que se incremente la opinión de que los mensajes de texto sustituyen a la comunicación formal.

\subsection{Se prefiere enviar un mensaje de texto a hacer una llamada}

Resultó inesperado que el porcentaje que actualmente prefiere enviar un mensaje de texto en vez de hacer una llamada es menor (73.8\%); esto es lo que me hace concluir que la tendencia de la preferencia a usar los mensajes de texto contra hacer una llamada es a la alza, que es lo que mencioné en el último párrafo de la conclusión anterior. Es importante concluir que esta tendencia se observa en todas las edades del grupo encuestado, tanto los mayores de 50 años, como los menores de 16 años.

Es importante comentar que en el grupo poblacional comprendido entre los 19 y 23 años, esta opinión se observa bien marcada puesto que entre los que prefieren enviar un mensaje (74\%) a los que piensan que si se está sustituyendo la comunicación formal por los mensajes de texto (88\%) se observa mucho su opinión respecto a esta tendencia. Se vuelve más relevante si consideramos que esta generación (mayormente estudiando en la universidad), es la que estará dentro de poco tiempo en puestos de decisión y manejando aún más la tecnología. Los datos muestran que esto se marca más aún con las generaciones más jóvenes.

\subsection{Respecto al SME más utilizado}

Podemos observar que el más utilizado es WhatsApp, con indicadores de Suma Ponderada Promedio (SPP) de 0.89, y elección en cualquier nivel de 32\%. Se pudo observar que al ver las elecciones en cualquier nivel, solamente 5 encuestados no indicaron preferir WhatsApp en ningún nivel.

Al revisar los comentarios dados, podemos concluir que la elección del SME se basa mucho en la rapidez, disponibilidad, capacidad para enviar fotos y videos y adicionalmente su costo. Otros comentarios fueron que lo prefieren porque cuando iniciaron su proceso de uso de SME, la mayoría de sus interlocutores ya tenían instalado el WhatsApp. Aunque la mayoría de los SME elegidos no tienen costos asociados directamente a costos por uso del SME (exceptuando los SMS y BBM) algunos encuestados mencionaron que si implica el costo de una conexión a internet que debe ser proporcionada por el proveedor de servicios móviles. 


\subsection{Preferencia de uso de fabricante en aparatos móviles}

Se pudo observar que en el mercado local, se utilizan más los aparatos de marca Samsung con una preferencia de uso del 36\% del total de los encuestados, el fabricante Apple (aparato móvil iPhone) es el segundo en el mercado con un 21\%. Los porcentajes se mantienen casi similares según los grupos poblacionales, por lo que se puede concluir que el uso del fabricante depende mucho de tres razones: a) facilidad de uso de su sistema operativo, b) precio del equipo móvil, y c) los aparatos ofrecidos por los Operadores de redes móviles en el país.

\subsection{Como utilizan los usuarios los SME}

Pude concluir que los usuarios de los sistemas de mensajería electrónica, utilizan los mensajes de texto principalmente para comunicarse con Amigos, con una suma ponderada de 423, con una preferencia en nivel 1 del 72\%; las observaciones siguientes arrojan que en segundo lugar los SME se prefieren para comunicación hacia familiares, que teniendo una suma ponderada de 232, su porcentaje de preferencia en nivel medio es de $52 \%$ y finalmente la preferencia en nivel bajo para uso de los SME fue para la comunicación con trabajo y estudio, que aun teniendo una suma ponderada de 256, el mayor valor de preferencia está en nivel bajo con $47 \%$.

\subsection{Recomendaciones}

Basado en la investigación realizada y las conclusiones obtenidas, se recomienda lo siguiente:

1. Ampliar la investigación respecto al marco legal o regulaciones legales respecto al uso inadecuado de los sistemas de mensajería electrónica.

2. Considerando el tiempo y la atención que brindan nuestros jóvenes a los SME, es importante para los sistemas de educación o procesos de enseñanza aprendizaje, que lo tomen en cuenta, con el fin de incorporarlos a estos procesos.

3. Es concluyente que la tendencia es a que cada vez más se usarán los sistemas de mensajería electrónica y aunado a lo anterior, a la era digital actual. Se recomienda hacer conciencia en todos los usuarios de los sistemas de mensajería electrónica a verificar si el interlocutor es realmente la persona que pensamos; puesto que siempre con el uso de los mensajes de texto o comunicaciones electrónicas, no hay una garantía total de que el interlocutor es realmente quien se piensa que es.

4. En muchos comentarios se pudo observar que la tendencia a usar más, los SME, se debe a la facilidad de enviar o recibir fotos o videos, pero esta es otra razón por la que debe tenerse la certeza de quien es nuestro interlocutor.

5. Al notar que los mensajes de texto se utilizan más para las comunicaciones entre amigos, se recomienda en el ámbito familiar, tratar de revertir estos resultados, tratando de que las comunicaciones vía cualquier SME, sean constantes e incluso sea una costumbre.

6. Ante todo lo anterior, considero que concluye en la despersonalización de las comunicaciones, donde no interesa tanto, las expresiones orales, las expresiones vocales sino transmitir el mensaje. Así que es notorio que este tipo de tecnología tiende a usarse y a mejorarse más con el tiempo, se recomienda que los desarrollos futuros se enfoquen en los aspectos de la seguridad de los usuarios, tanto en la 
información personal como en dar la mayor seguridad posible de quien es nuestro interlocutor.

\section{Bibliografía}

Crespo, Gonzalo O. (01 de Junio del 2014). Los Niños de Hoy, Nativos Digitales. Recuperado 11 de Agosto de 2014, del sitio web del Diario de Hoy, Ecuador: http://www.hoy.com.ec/noticias-ecuador/los-ninos-de-hoy-nativos-digitales607455.html

Dannenfeldt, Diana (2006). How BackBerry Messenger Works. Recuperado el 28 de Julio de 2014 del sitio http://computer.howstuffworks.com/e-mailmessaging/blackberry-messenger3.htm

Fuller, Wayne A. (Agosto 2009). Sampling Statistics. Publisher: John Wiley \& Sons. Pags $3-20$.

Horn, Jeff, Rosenband, Leonard N., \& Smith, Merritt Roe. (Octubre 2010) Dibner Institute Studies in the History of Science and Technology: Reconceptualizing the Industrial Revolution. MIT Press.

ITU: International Telecommunications Union (2011) The World in 2010. Place des Nations, 1211 Geneva 20 - Zwitzerland.

Levine, David M., Krehbiel, Timothy C., Berenson, Mark L. (Cuarta Edición. 2006) Estadística para Administración. Pearson. (pag. 256 - 257)

Palfrey, John \& Gasser, Urs. Born Digital (2008), Understanding the first generation of digital natives. Basic Books.

Santos, I. (10 de Junio de 2014). Recuperado el 20 de Julio de 2014. WhatsApp. Del sitio: http://es.wikipedia.org/wiki/WhatsApp.

Triolla, Mario F. (Décimo primera edición. 2013) Estadística. Pearson Education, (pag. 328 - 337) ISBN: 9786073217682.

Universidad de Lomas de Zamora. (Diciembre de 2010). Reporte Macroeconómico Diciembre 2010. Facultad de Ciencias Económicas, Instituto de Investigaciones Económicas.

\footnotetext{
"LA REVISTA INNOVARE NO SE HACE RESPONSABLE EN NINGÚN CASO DE LOS CONTENIDOS, DATOS, CONCLUSIONES U OPINIONES VERTIDAS EN LOS ARTÍCULOS PUBLICADOS, SIENDO ESTA RESPOSABILIDAD EXCLUSIVA DEL (DE LOS) AUTOR (AUTORES)”
} 